\title{
The Long Wavelength Array
}

\section{Ylva Pihlström*}

University of New Mexico, USA

E-mail: ylva@unm.edu

The Long Wavelength Array (LWA) will be a new, open-skies, user-oriented aperture synthesis instrument dedicated to explore frequencies between 20 and $80 \mathrm{MHz}$. The LWA will provide high temporal (millisecond or better) and high spatial resolution (arcsecond) and mJy-level sensitivity. The LWA key science areas include acceleration, propagation, and turbulence in the ISM; the high-redshift Universe; planetary, solar and space science; and the transient universe at radio wavelengths. In addition, key goals of the LWA are as a training ground for the next generation of radio astronomers and to re-invigorate radio astronomy in the US at the university level. The LWA will be operated by the University of New Mexico on behalf of the South West Consortium (SWC), thereby providing opportunities for students within the fields of astronomy, computer science and electrical engineering. Currently, in its first year of construction funding, the LWA team is now bringing up the first station near the Very Large Array site in the southwest US.

The 8th European VLBI Network Symposium

September 26-29, 2006

Toruń, Poland

${ }^{*}$ Speaker. 


\section{Introduction}

Compared to wavelengths short-ward of about $20 \mathrm{~cm}$, at longer wavelengths, the Universe is poorly explored. Thus, many astrophysical problems remain to be investigated and discovered at the lowest end of the radio spectrum. To develop a usable long-wavelength telescope, the Naval Research Lab (NRL) and the National Radio Astronomy Observatory (NRAO) worked together to add a 74-MHz system to the Very Large Array (VLA). Scientific promise at low radio frequencies has been demonstrated by the successful completion of this system, which has been available to a growing user community since 1998 . With the VLA $25^{\prime \prime}$ angular resolution, a few radio sources have been resolved spatially, yielding interesting and pioneering work in, for instance, the physics of supernova remnants and particle acceleration (e.g. [1]). More importantly, the VLA 74-MHz system has shown that the development of self-calibration techniques and wide-field imaging lifts the constraints that for a long time confined long-wavelength astronomy to poor angular resolution and consequently confusion-limited sensitivities. It should be noted that the VLA was never designed to operate at such low frequencies, resulting in mediocre $74-\mathrm{MHz}$ system efficiencies and thus, at best, sensitivities of $25 \mathrm{mJy} /$ beam. To reach more ambitious science goals, resolutions of a few arcseconds and sensitivities of $<1 \mathrm{mJy}$ are desirable. This can only be achieved by a specifically designed instrument dedicated to operate at low frequencies, illustrating the need for the Long Wavelength Array (LWA) as well as other low-frequency instruments such as the Mileura Widefield Array (MWA) and the Low Frequency Array (LOFAR).

\section{LWA design concept}

The LWA will be an open-skies, user-oriented aperture synthesis instrument designed to operate between 20 and $80 \mathrm{MHz}(15-4 \mathrm{~m})$, a range where the ionosphere limited previous instruments to $\sim 5 \mathrm{~km}$ baselines. The demonstration by the 74-MHz VLA system that this limit can be overcome, inspired development of the 400-km LWA that will achieve unprecedented spatial resolution ( $2-8^{\prime \prime}$, depending on frequency) and sub-mJy sensitivity, i.e. 2-3 orders of magnitude improvement over past capabilities. Together with high temporal (millisecond or better) resolution, the LWA is therefore well suited to probe the transient Universe at long wavelengths where previous undiscovered classes of emitters are hinted at from low-resolution VLA observations.

A full description of the array design is beyond the scope of this presentation, however a brief overview $^{1}$ of the LWA is necessary to illustrate the technical and hence the scientific capabilities of the instrument. At the top hierarchical level, the complete LWA will consist of 52 stations spread across the state of New Mexico (Fig. 1) in a pattern designed to provide optimal u-v coverage, in turn optimizing the imaging and calibration parameters. A single station consists of 256 linearly polarized crossed dipole elements distributed over a 100-m diameter area. Each station will be phased up as an individual beam using electronic phase-delay adjustments, to be correlated with the other stations. Given that each dipole can see the entire sky and the beam is steered electronically, the beam can be pointed to any position in the sky without the involvement of any mechanically moving parts. The electronic beam steering also means that multiple beams can be formed simultaneously and directed independently anywhere on the sky, enabling a very flexible observing

\footnotetext{
${ }^{1}$ A more detailed description can be found at http://lwa.unm.edu
} 


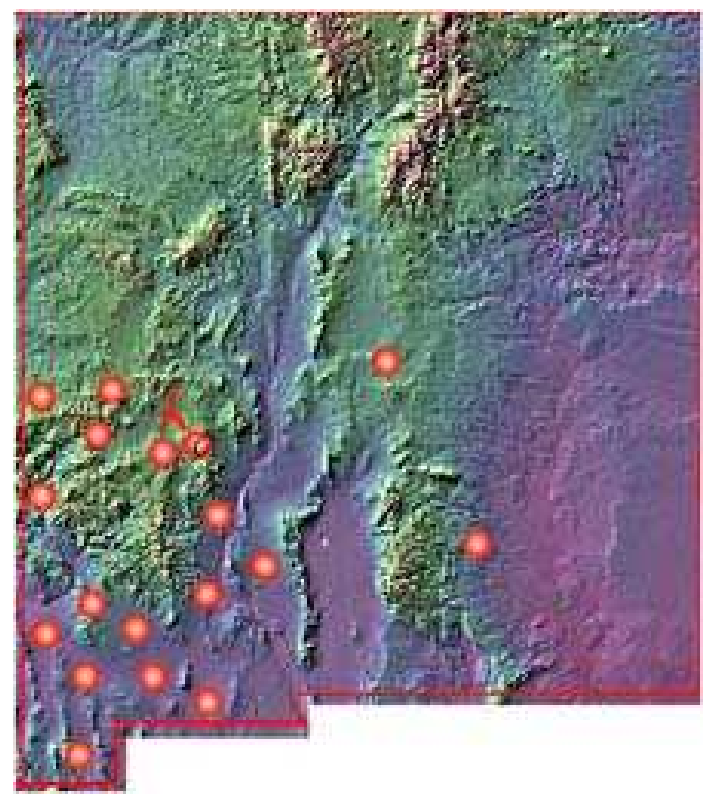

Figure 1: LWA stations will be spread throughout and possibly beyond the state of New Mexico. The upside-down Y-symbol denotes the location of the VLA.

instrument with many potential operation modes. The number of stations is mainly determined to achieve sufficient collecting area while the number of dipole elements per station is determined by the signal-to-noise ratio required to perform self-calibration.

\section{LWA scientific and educational goals}

The LWA will explore the transient universe at long wavelengths where previous undiscovered classed of emitters, including steep-spectrum sources are expected. The LWA science areas include: 1) acceleration of relativistic particles in, for example, supernova remnants, radio galaxies and clusters, and ultra-high energy cosmic rays; 2) cosmic evolution and the high-redshift Universe, including evolution of dark matter and dark energy by differentiating relaxed and merging clusters, and studying the first black holes; 3) plasma astrophysics and space science, including the acceleration, propagation and turbulence in the ISM of the Milky Way and normal galaxies; and 4) the transient Universe including magnetar giant flares, gamma-ray bursts and new sources of unknown origin. To undertake the LWA project, the South West Consortium (SWC) was created, consisting of the University of New Mexico (UNM) as the lead institute, the Naval Research Lab (NRL), Los Alamos National Laboratories (LANL) and the Applied Research Labs at University of Texas at Austin (ARL-UT).

In addition to the scientific goals, other important goals of the LWA are as a technical training ground for the next generation of radio astronomers and to re-invigorate radio astronomy in the US at the university level. Great discoveries in astronomy are often enabled by technological advancements, for instance in receiver development or improvements of calibration algorithms. This illustrates the importance of an all-embracing understanding of the field as the science and engineering communities have naturally evolved into more specialized, and separate communities 
which may lead to a weakening of the communication between the disciplines. The LWA requires a new approach to software and hardware, and therefore serves as a pathfinder for larger international low-frequency telescope projects like the Square Kilometer Array (SKA). Much of this work will be performed at US universities, providing training for the next generation of radio astronomers and telescope designers. At UNM, the LWA will provide a unique opportunity to intermix research and development at a student level. Student projects will include design, fabrication, RFI mitigation, observing, software development, and development of imaging and calibration algorithms.

To engage the LWA as both a means of providing technical training of students, and to pursue excellent science at the university level, four university-based scientific testing and evaluation teams have been set up. These teams will provide early feedback from scientific experiments, thereby allowing the possibility to optimize the final LWA. The four teams will focus on the following four research areas respectively: 1) high-resolution imaging and particle acceleration; 2) wide-field imaging and cosmic evolution; 3) ionosphere and ionospheric physics; and 4) RFI mitigation and excision, and transient events.

\section{A phased construction}

To ensure that the scientific objectives will be met for the final 52 station array, the LWA will be built in phases. While it is the goal of the LWA to produce science in every phase, this will also allow feedback from scientists and hence the possibility to improve the instrument into the next phase of the construction. Table 1 outlines the timescale of the planned construction phases. Phase 0 is the now completed 74-MHz VLA system which demonstrated the technical feasibilities of a low-frequency instrument. Currently, the LWA project is in Phase I: the construction of the Long Wavelength Demonstrator Array (LWDA) station in addition to the first LWA station. The LWDA station differs slightly from the remaining LWA stations being a scaled-down $(60-80 \mathrm{MHz})$ version optimized to function well with the VLA 74-MHz system. In Phase I, the aim is to test and develop the structural design of antennas and receivers to determine the final design. In addition, we will work towards incorporating LWA stations into the VLA 74-MHz system.

\begin{tabular}{|c|c|c|c|}
\hline Time & Phase & Description & Status \\
\hline 2004 & 0 & 74-MHz system at VLA & Completed \\
\hline 2006-2008 & $\mathrm{I}$ & $\begin{array}{l}\text { Long Wavelength Demonstrator Array } \\
\text { + LWA station } 1\end{array}$ & $\begin{array}{l}\text { Funded, and } \\
\text { construction started }\end{array}$ \\
\hline $2007-2010$ & II & 9 station array & \\
\hline 2010-2012 & III & LWA core & \\
\hline 2012-2014 & IV & High-resolution LWA & \\
\hline 2010- & IV & LW operations and science centre & \\
\hline
\end{tabular}

Table 1: Time line for the construction phases of the LWA, as of October 2006

The LWDA station is located next to the VLA which facilitates setting up our operations given that an infrastructure already exists. To date, we have installed the first 16 antenna structures at the LWDA site (Fig. 2). During 2006, receivers will be installed at the site and an intense testing period will take place. The antenna structure and design, as well as the receive chain design and functionality will be evaluated before manufacturing of a larger array will begin. 


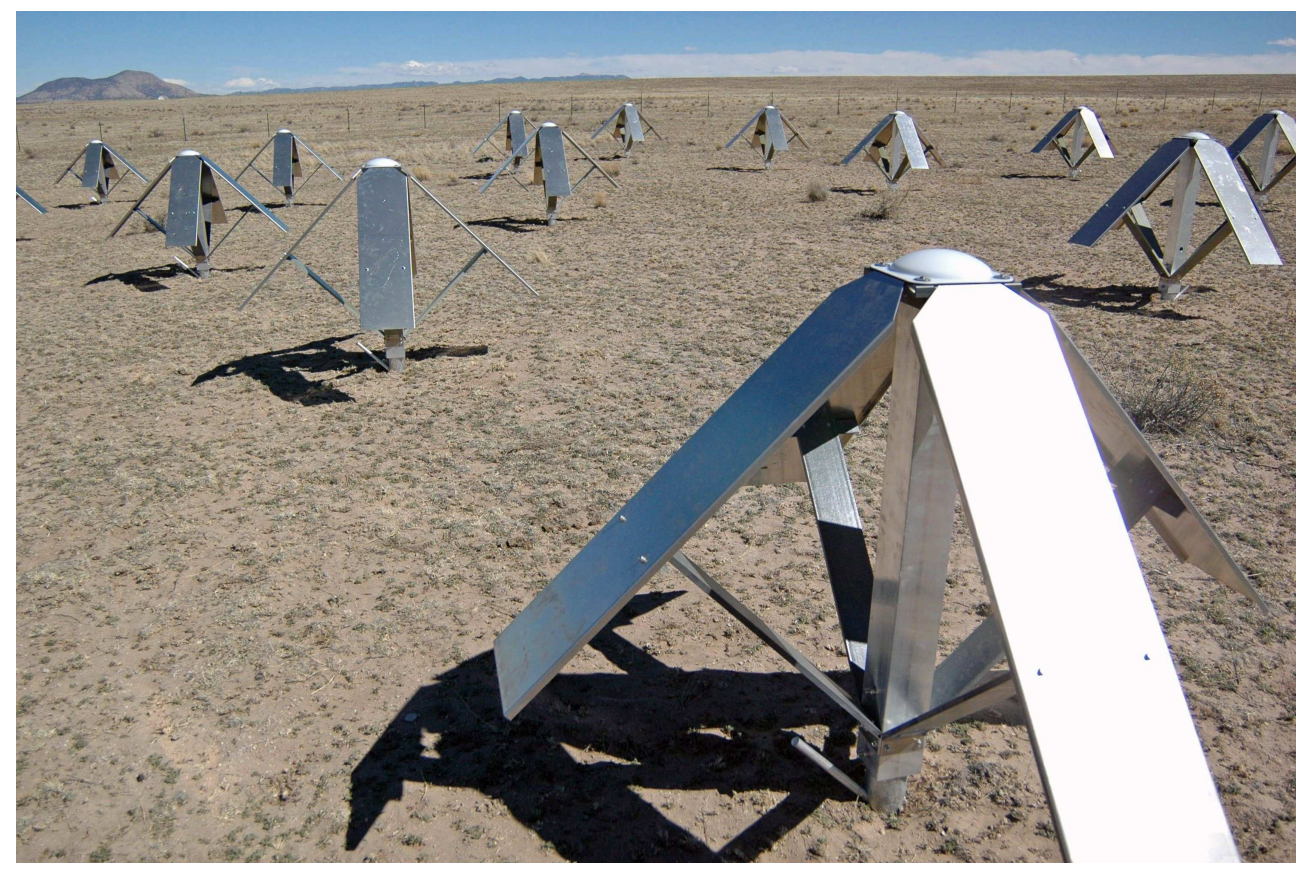

Figure 2: The first 16 installed dipole antennas at the LWDA station located next to the VLA.

\section{References}

[1] Brogan, C.L., Lazio, T.J., Kassim, N.E., \& Dyer, K.K., 2005, AJ, 130, 148 